\title{
Difference-Histogram Modification based on Reversible Data Hiding
}

\author{
Neelima Dadsena \\ Electronics \& Telecommunication Engineering \\ Chhattrapati Shivaji Institute of Technology, Durg
}

(C.G.) India

\begin{abstract}
Reversible or lossless data embedding is a method that embeds information into an image in a reversible behavior. The histogram alteration based reversible data hiding method using causal window is planned which predicts the embedding level with the help of the pixel significance, edge significance. Using this data embedding level the data is embedded into the pixels. The pixel level modification considering the human visual system characteristics is also made to decrease the distortion caused by data embedding. This drastically improves the information embedding capability along with greater visual quality. The experiment outcome and performance comparison are presented to express the validity of the proposed algorithm.
\end{abstract}

\section{Keywords}

Pixel-Pair-Mapping (PPM), Reversible Data Hiding (RDH), Difference-Pair-Mapping (DPM), EC (Embedding Capacity).

\section{INTRODUCTION}

In recent era the use of information technology and internet had been growing rapidly, so the use of digital information such as image, audio, video and text are communicated and share very conveniently. Due to easy access of internet the data can be easily copied forged and modified. Therefore, information security is severe deficient and the forger tries to violet the content of the multimedia. Hence, for the protection of the originality and imperceptibility of the multimedia data, the secret message must be hidden in the image. Data hiding is a technique to conceal secret data into cover object, which plays a vital role in the protection of the information [1].

Mainly in the cases of data hiding, the cover object will affected by some distortion and cannot be reversed back to the original object, because of some stable distortion has been occurred to the cover object even after the extraction of hidden message. Reversible data hiding facilitates vast possibility of applications to link two sets of data, in that manner that the hidden message have been extracted out without effecting the cover object. Hence, provide an additional possibility for handling two different data sets.

Many reversible data hiding schemes have been proposed. Tian [9] proposed a difference expansion data hiding scheme, where the difference and average values of two neighboring pixels are calculated and the secret data to be embedded are appended to a difference value represented as a binary number. Alattar [4], Kim et al [5], and Weng [7] further extended Tian's work. Ni et al. proposed a scheme of using peak/zero points in the histogram of spatial domain images. Fridrich et al [10] proposed several methods to embed data. The main idea of their work is to compress the selected image features for acquiring spare space. Hong et al. [2] presented a scheme which performs a shift of the histogram of prediction errors. It use the median edge detector (MED) to predict pixel values. Barton [3] developed a reversible data embedding

\author{
Archana Tiwari \\ Electronics \& Instrumentation Engineering \\ Chhatarpati Shivaji Institute of Technology, Durg \\ (C.G.) India
}

algorithm that is based on data compression. In this method compressed data is to be embedded in an image.

This paper present a difference histogram modification reversible data hiding algorithm. In this proposed method the pixel pair context is convenient in projecting a local image region on two dimensional space for accomplishing a sequence that consist of difference pairs. Now by counting the difference pairs a two dimensional difference histogram is generated. As the DPM is an injective mapping technique which is defined on difference pairs and it is used in recent histogram based methods by natural extension of expansion embedding and shifting techniques. So finally by difference pair mapping (DPM) technique reversible data embedding is implemented.

For enlightening the embedding performance the proposed method uses two dimensional difference histogram and its specific DPM, as compared with the conventional one dimensional histogram based methods which include more pixels for carrying the data and also we can reduce the number of shifted pixels [8]. In the previous literature studies of embedding position and selection techniques a new pixel pair selection strategy is proposed for locating the pixel pairs in smooth image regions to embed the data. Also, it is further used for enhancing the embedded performance.

In present paper reversible data hiding method by using a two-dimensional difference histogram are discussed for covert communication. In RDH algorithm original information can be completely recovered. In section 2 the proposed $\mathrm{RDH}$ scheme is introduced and discussed, section 3 discusses the experimental results of proposed method and previous methods. finally section 4 presents brief conclusion of paper.

\section{PROPOSED RDH SCHEME}

The proposed RDH scheme is based on modification of two dimensional difference histogram by constructing a DPM which is an injective mapping defined on difference pairs. For a pixel pair $(\mathrm{x}, \mathrm{y})$, compute two difference values $\mathrm{d}_{1}=\mathrm{x}-\mathrm{y}$ and $d_{2}=y-z$ to form a two dimensional difference histogram of $\left(\mathrm{d}_{1}, \mathrm{~d}_{2}\right)$, where $\mathrm{z}$ is a prediction of $\mathrm{y}$. The pixel pair $(\mathrm{x}, \mathrm{y})$ has four choices : $(\mathrm{x}-1, \mathrm{y}),(\mathrm{x}+1, \mathrm{y}),(\mathrm{x}, \mathrm{y}-1)$, or $(\mathrm{x}, \mathrm{y}+1)$ (see fig. 1(a)). Based on these four modification directions, Lee et al.'s method can be improved by designing a new PPM [15].

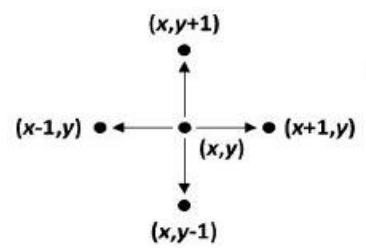

(a)

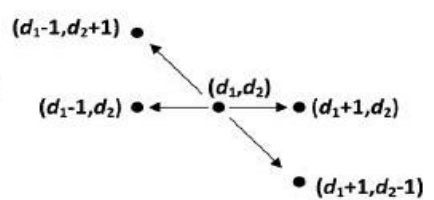

(b)

Fig. 1(a) By modifying either $x$ or $y$ by $1,(x, y)$ has four modification directions. (b) The corresponding difference pair also has four modification. 
Inspired by the above mentioned new PPM, either $\mathrm{x}$ or $\mathrm{y}$ is modified by 1 . In the PPM, since (x, y) has four modification directions.

The difference pair also has four modifications directions : $\left(\mathrm{d}_{1}-1, \mathrm{~d}_{2}\right),\left(\mathrm{d}_{1}+1, \mathrm{~d}_{2}\right),\left(\mathrm{d}_{1}+1, \mathrm{~d}_{2}-1\right),\left(\mathrm{d}_{1}-1, \mathrm{~d}_{2}-1\right)$, given in fig. $1(\mathrm{a})$ and 1(b). For example, by modifying to, the modification direction to $(\mathrm{x}, \mathrm{y})$ is "up" and the corresponding alteration direction to $\left(d_{1}, d_{2}\right)$ is "upper-left", since $d_{1}$ changes to $d_{1}-1$ and $d_{2}$ changes to $d_{2}+1$. Based on these four modification directions, a new RDH method by designing a DPM is introduced. The idea of two-dimensional pixel intensity histogram of Lee et al., is complete to two dimensional difference histogram. Besides, for each $(x, y)$, compute the prediction of $\mathrm{y}$ based on the context of $(\mathrm{x}, \mathrm{y})$ for an accurate estimation. Here, the gradient adjusted prediction (GAP) will be used in this scheme.

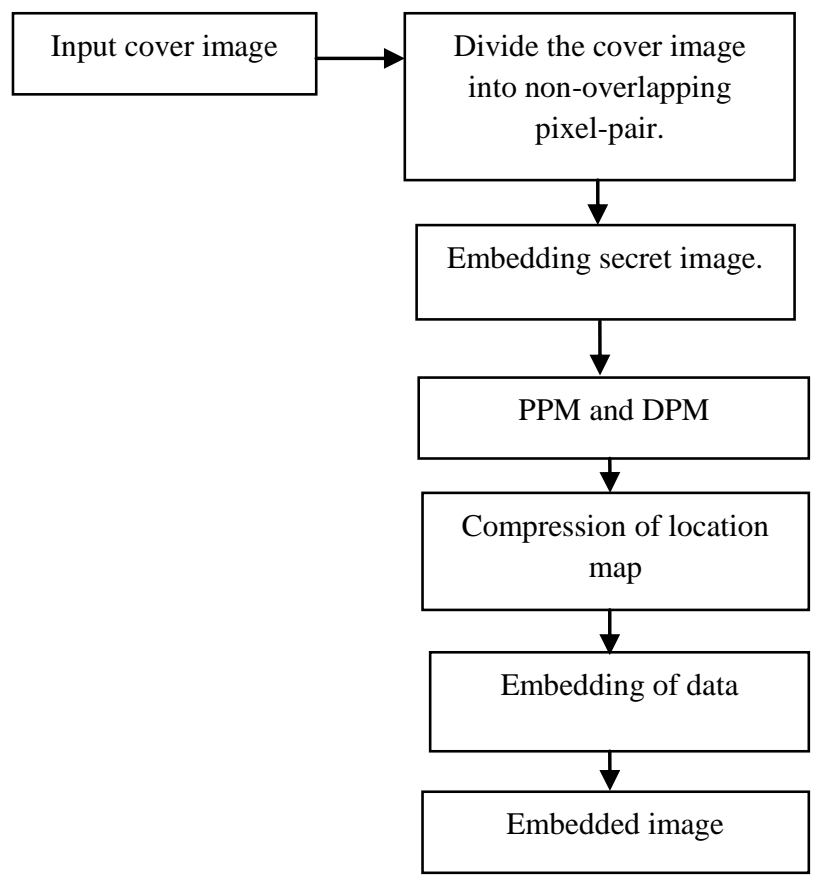

Figure. 2. Block diagram for embedding procedure

The brief introduction of the embedding method is given below. Fig. 2 shows the block diagram of embedding procedure. Initially the cover image is partitioned into non overlapping pixel-pairs. Then that secret message is embedded into a part of cover image represented as I'. For getting a binary sequence record the least significant bits (LSB) of some pixels which is represented as I', and embed this sequence into the rest part of I, i.e., I-I'. At last to embed the auxiliary information and the compressed location map into I" using LSB replacement is used. To overcome the overflow and underflow problems overflow location map is used and compression of location map is achieved by arithmetic coding. By DPM method it can be embed the secret data while by LSB replacement method auxiliary data is embedded. The smooth pixel pairs are used to embed the secret data in the embedding technique which can enhance the performance also. Both data extraction procedure and data embedding procedure are reverse in nature. In data extraction procedure, first the LSB replacement is performed to extract the compressed location map and then location map is generated by decompressing it. While decompression of the location map is done by blind decoding. The prediction and noisy level are computed according to the equation (1) and
(2). The image restoration can be realized by extracting the LSB sequence.

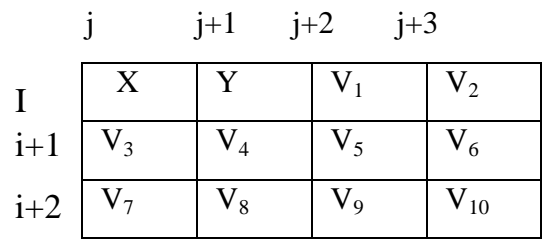

Fig. 3. Context of $(x, y)$, where the location of pixel $x$ is (i, j).

Here, in fig. 3 the blue pixels are taken for the computation of the GAP predictor for $\mathrm{y}$, and remaining pixels $\left\{\mathrm{v}_{1}, \ldots . \mathrm{v}_{10}\right\}$ will use for the computation of the noisy level.

$$
\mathrm{Z}=\left\{\begin{array}{cl}
v_{1}, & \text { if } d_{v}-d_{h}>80 \\
\frac{\left(v_{1}+u\right)}{2}, & \text { if } d_{v}-d_{h} \in(32,80] \\
\frac{\left(v_{1}+3 u\right)}{4}, & \text { if } d_{v}-d_{h} \in(8,32] \\
u, & \text { if } d_{v}-d_{h} \in[-8,8] \\
\frac{\left(v_{4}+3 u\right)}{4}, & \text { if } d_{v}-d_{h} \in[-32,8) \\
\frac{\left(v_{4}+u\right)}{2}, & \text { if } d_{v}-d_{h} \in[-80,-32) \\
v_{4}, & \text { if } d_{v}-d_{h}<-80
\end{array}\right.
$$

Where $\left\{\mathrm{v}_{1}, \ldots \ldots \mathrm{v}_{7}, \mathrm{v}_{8}\right\}$ are neighboring pixels of $(\mathrm{x}, \mathrm{y})$ (see fig. $2), d_{v}=\left|v_{1}-v_{5}\right|+\left|v_{3}-v_{7}\right|+\left|v_{4}-v_{8}\right|$ and $d_{h}=\left|v_{1}-v_{2}\right|+\left|v_{3}-v_{4}\right|$ $+\left|v_{4}-v_{5}\right|$ represent the vertical and horizontal gradients, and $u$ $=\left(\mathrm{v}_{1}+\mathrm{v}_{4}\right) / 2+\left(\mathrm{v}_{3}-\mathrm{v}_{5}\right) / 4$. Notice that $\mathrm{z}$ should be rounded to its nearest integer if it is not an integer. Then, compute the noisy level of $(\mathrm{x}, \mathrm{y})$ denoted as using its ten neighboring pixels as $\mathrm{NL}(\mathrm{x}, \mathrm{y})$ using its ten neighboring pixels $\left\{\mathrm{v}_{1}, \ldots \ldots \mathrm{v}_{7}, \mathrm{v}_{8}\right\}$ as

$$
\mathrm{NL}=\int_{\left(i^{\prime}, j^{\prime}\right) \varepsilon v}\left|\nabla I\left(i^{\prime}, j^{\prime}\right)\right|
$$

Where $\mathrm{V}$ represents the context of containing the ten pixels and $\nabla$ stands for the gradient operator. Here, for discrete image, the noisy level is computed by summing both vertical and horizontal differences of every two consecutive pixels in $\mathrm{V}$, and it is less than or equal to $13 \times 255=3315$. Clearly a pixel pair located in smooth regions may have a small noisy level. Finally for each pixel pair with noisy level less than a threshold $\mathrm{T}$, compute the difference pair $\left(\mathrm{d}_{1}, \mathrm{~d}_{2}\right)$ and implement data embedding.

\section{EXPERIMENTAL RESULTS}

Six 512X512 sized gray scale images including Lena, Boat, Airplane, Bird, and Rice are used, shown in fig 4 . The proposed method is superior to abovementioned methods [14]. It experimentally demonstrates that the DPM based scheme can provide a much better performance than PPM.

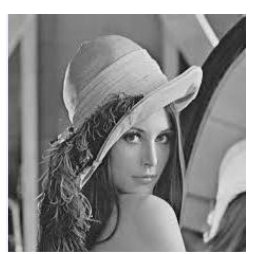

(a) Lena

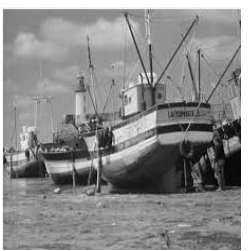

(b) Boat

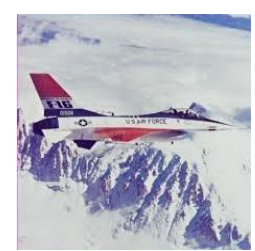

(c) Airplane 


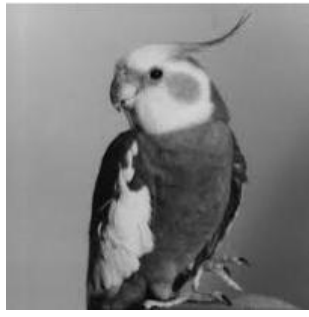

(d) Bird

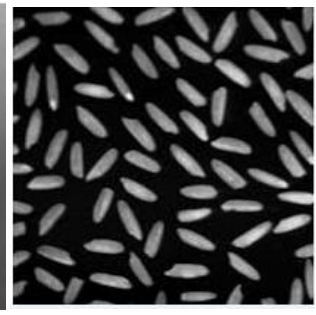

(e) Rice
Fig. 4. The experimental grayscale image

The performance of the proposed data hiding scheme on different images has been evaluated. The PSNR value of Lena is good comparing for other images.

Table I. Comparison of proposed method and other method

\begin{tabular}{|c|c|c|c|c|c|c|}
\hline Algorithm & Items & Lena & Boat & $\begin{array}{c}\text { Air- } \\
\text { plane }\end{array}$ & Bird & Rice \\
\hline \multirow{2}{*}{$\begin{array}{c}\text { Hong et } \\
\text { al's }\end{array}$} & $\begin{array}{c}\text { EC } \\
\text { (bits) }\end{array}$ & 16640 & 15000 & 12640 & 16230 & 14340 \\
\cline { 2 - 7 } & PSNR & 48.81 & 48.32 & 48.24 & 48.73 & 48.80 \\
\hline \multirow{2}{*}{ Ni et al's } & $\begin{array}{c}\text { EC } \\
\text { (bits) }\end{array}$ & 16640 & 15000 & 12640 & 16230 & 14340 \\
\cline { 2 - 7 } & PSNR & 48.73 & 48.45 & 48.33 & 49.01 & 48.84 \\
\hline \multirow{2}{*}{ Proposed } & $\begin{array}{c}\text { EC } \\
\text { (bits) }\end{array}$ & 16640 & 15000 & 12640 & 16230 & 14340 \\
\cline { 2 - 7 } & PSNR & 49.10 & 48.93 & 48.91 & 48.77 & 48.90 \\
\hline
\end{tabular}

The performance comparisons of PSNR between the proposed method and other methods are shown in table I. The hiding payload of the proposed method is higher than the other methods. The PSNR values are also higher than other method. The hiding payload and image quality of the proposed are significantly achieved to good performances.

\section{CONCLUSION}

This proposed work, focus on a new reversible data compression hiding scheme for hiding message in JPEG image. In this to location map will be compressed to increase in order to increase the embedding capacity. The location map depend on the payload or hidden message. Comparing with other RDH techniques and literature, this present work often has enhanced flexibility to different images and larger embedding capacity for the same image quality.

\section{ACKNOWLEDGMENT}

I am very grateful to the Chhattrapati Shivaji Institute of Technology, Durg for giving me such a pleasant environment to learn \& also want to thank my guide Mrs. Archana Tiwari for providing me the necessary support for this research.

\section{REFERENCES}

[1] Zhenxing Qian, Xinpeng Zhang and ShuozhongWang, "Reversible Data Hiding in Encrypted JPEG Bitstream." IEEE Trans. on Multimedia, VOL. 16, NO.5, Apr. 2014.

[2] W. Hong, T. S. Chen, and C. W. Shiu, " Reversible Data Hiding Based on Histogram Shifting of Prediction Errors," in Intelligent Onformation Technology Application Workshops, pp. 292-295, 2008.
[3] J.M. Barton, "Method and Apparatus for Embedding Authentication Information Within Digital Data," U. S. Patent 5646997, 1997.

[4] A. M. Alattar, " Reversible Watermark Using the Difference Expansion of a generalized Integer Transform, IEEE Transaction Image processing, VOL. 13, no. 8 pp. 1147-1156, 2004.

[5] H. J. Kim, V. Sachnev, Y. Q. Shi, J. Nam, and H. G. Choo, A Novel Difference Expansion Transform for Reversible Data Embedding, IEEE Transaction Information Forensics and Security, vol. 3, no. 3, pp. 456-465, 2008

[6] M. Fallahpour, "Reversible image data hiding based on gradient adjusted prediction," IEICE Electron. Express, vol. 5, no. 20, pp.870-876, Oct. 2008.

[7] S. W. Wang, Y. Zhao, and J. S. Pan, "A Novel Reversible Data Hiding Scheme," International Journal of Innovative Computing, Information and control, vol. 4, no. 2, pp. 351-358, 2008.

[8] Xinpeng Zhang, "Separable Reversible Data Hiding in Encrypted Image." IEEE Trans. on information forensics and security, VOL. 7, NO.2, Apr. 2012.

[9] J. Tian, "Reversible Data Embedding Using a Difference Expansion" IEEE Transaction Circuits Syst. Video Technology VOL. 13, NO.8, Aug. 2003.

[10] J. Fridrich, M. Goljan, and R. Du, "Lossless data Embedding - New Paradigm in Digital Watermarking," EURASIP J. Application Signal Process., vol. 2002, no. 2, pp. 185-196, Feb 2002.

[11] Peyman Rahmani, Ehsan Rahmani and Gholamhossein Dastghaibyfard, "A Location Map Free Reversible Data Hiding Scheme for VQ- Compressed Images Based on Search Order Coding." Seventh International Conference on Intelligent Information Hiding and Multimedia Signal Processing, 2011.

[12] Ching-Te-Wang, Ching-Lin Wang, Lin-Chun Li and Sheng-You Guo, "The Image Capacity and Reversible Data Hiding Technique Based on Pixel Frequency of Block.” IEEE 2011.

[13] Peyman Rahmani and Ehsan Rahmani, "A Reversible Data Embedding Scheme Based on Search Order Coding for VQ Index Tables." International ISC conference on information Security and cryptology (ISCISC), Sept 14$15,2011$.

[14] X. Gao, L. An, Y. Yuan, D. Tao, and X. Li, "Lossless Data Embedding Using Generalized Statistical Quantity Histogram," IEEE Trans. Circuits Syst. Video Technol., vol. 21, no. 8, pp. 1061-1070, Aug. 2011.

[15] W. L. Tai, C. M. Yeh, and C. C. Chang, "Reversible data hiding based on histogram modification of pixel differences," IEEE Trans. Circuits Syst. Video Technol., vol. 19, no. 6, pp. 906-910, Jun. 2009.

[16] X. Li, B. Yang, and T. Zeng, "Efficient Reversible Watermarking Based on Adaptive Prediction Error Expansion and Pixel Selection," IEEE Trans. Image Process., vol. 20, no. 12, pp. 3524-3533, Dec. 2011. 